\title{
Le véhicule autonome : se désengager et se réengager dans la conduite
}

The autonomous vehicle: disengagement and reengagement

\section{Jean-Baptiste Haué, Sophie Le Bellu et Cécile Barbier}

\section{(2) OpenEdition}

\section{Journals}

Édition électronique

URL : http://journals.openedition.org/activites/4987

DOI : 10.4000/activites.4987

ISSN : 1765-2723

\section{Éditeur}

ARPACT - Association Recherches et Pratiques sur les ACTivités

\section{Référence électronique}

Jean-Baptiste Haué, Sophie Le Bellu et Cécile Barbier, « Le véhicule autonome : se désengager et se réengager dans la conduite», Activités [En ligne], 17-1 | 2020, mis en ligne le 15 avril 2020, consulté le 17 avril 2020. URL : http://journals.openedition.org/activites/4987 ; DOI : https://doi.org/10.4000/ activites. 4987

Ce document a été généré automatiquement le 17 avril 2020

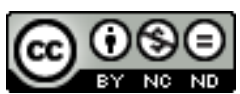

Activités est mis à disposition selon les termes de la licence Creative Commons Attribution - Pas d'Utilisation Commerciale - Pas de Modification 4.0 International. 


\section{Le véhicule autonome : se désengager et se réengager dans la conduite}

The autonomous vehicle: disengagement and reengagement

Jean-Baptiste Haué, Sophie Le Bellu et Cécile Barbier

\section{NOTE DE L'ÉDITEUR}

Article soumis le 12 décembre 2018, accepté le 17 février 2020

Les auteurs remercient Germain Poizat pour sa relecture constructive de l'article.

\section{Introduction}

1 Les échos médiatiques des sorties puis accidents provoqués par les véhicules autonomes de Google, Tesla, Uber et Apple, depuis février 2016, ont mis en lumière de manière spectaculaire le projet de véhicule sans conducteur tout en accentuant l'ensemble des promesses, angoisses, et fantasmes qui l'accompagnent. Les acteurs du monde automobile réorientent leurs efforts et développements sur une étape intermédiaire, plus plausible dans un futur proche : celle d'un véhicule doté de niveaux d'autonomie croissants, initié depuis l'arrivée sur le marché des premières assistances à la conduite - régulateur de vitesse, régulateur de distance adaptatif, puis plus récemment d'aide au parking, aide au maintien dans la voie, etc.

2 Le niveau $4^{1}$ décrit ainsi un stade d'automatisation du véhicule dans lequel le conducteur est encore à sa place, mais libéré des contraintes de maintien du volant / des pédales et de surveillance de la route. Si le conducteur accepte ce "lâcher-prise ", ce qui suppose la construction d'une confiance (Lee \& See, 2004) et l'inhibition des automatismes, routines et habitudes de conduite appris et profondément appropriés, il 
peut alors se consacrer à des activités dites de vie à bord, qui permettent de mettre à profit le temps disponible. Ce détachement de la conduite aura néanmoins pour probable conséquence de rendre plus difficile le retour à la conduite manuelle, autrement appelée reprise en main (voir par exemple une revue d'étude par De Winter, Happee, Martens, \& Stanton, 2014). Un tel paradoxe de l'automatisation a été identifié bien avant l'arrivée du véhicule autonome par Bainbridge (1983), qui décrit comment l'amélioration de la performance nominale d'un système amène de nouveaux problèmes lors du retour à la conduite manuelle dans une logique dite d'" effet bûcheron " ("Lumberjack effect") : plus l'arbre est haut, plus il tombe loin ${ }^{2}$; plus le niveau d'automatisation est important, plus les performances de la reprise en main par le conducteur se dégradent (Onnasch, Wickens, Li, \& Manzey, 2014).

3 Les études portant sur les épisodes de reprise en main sont récentes dans le milieu automobile puisque les véhicules présentant un niveau d'automatisation élevé sont encore à l'étude sous forme de prototypes. La littérature fait état de propositions de taxonomies relatives aux différents niveaux d'automatisation et aux transitions qui les lient (Lu, Coster, De Winter, 2016 ; SAE, 2018). Elle rapporte des études portant sur la mesure des temps de reprise en main (Dogan, Rahal, Deborne, Delhomme, Kemeny, \& Perrin, 2017 ; Gold, Damböck, Lorenz, \& Bengler, 2013 ; Zhang, Winter, Varotto, \& Happee, 2019) ou encore sur l'incidence de l'automatisation sur la Situation Awareness du conducteur (De Winter et al., 2014; Gibson, Lee, Venkatraman, Price, Lewis, Montgomery et al., 2016; Onnasch et al., 2014). Toutefois, à l'exception des travaux relatifs à la Situation Awareness (SA), pas ou peu de travaux de recherche abordent la reprise en main sous l'angle de l'activité elle-même, éclairant le vécu de la transition par le conducteur. La description du point de vue de l'utilisateur permet pourtant d'identifier les dimensions structurantes de son activité sans dépendre d'a priori théoriques.

4 L'objectif de cet article est précisément de documenter les processus de désengagement et de réengagement perceptivo-moteur et cognitifs du conducteur lors de la conduite d'un véhicule autonome de niveau 4, et ceci dans la perspective (1) de fournir des recommandations pour guider la conception centrée-utilisateur du cockpit d'un véhicule autonome, et (2) de préciser conceptuellement la notion d'engagement largement mobilisée dans les recherches sur la conduite autonome. Le phénomène de retour à la conduite manuelle après une phase de conduite autonome sera d'abord défini comme une activité située (Suchman, 1987) et distribuée (Hutchins, 1995b; Hutchins \& Klausen, 1996). Il sera ensuite décrit comme un réengagement de l'activité dans le monde de la conduite grâce aux notions du Cours d'Action (section 2). L'analyse fine d'un cas d'étude empirique nous aidera à définir ce que signifie se remettre dans la boucle de l'activité de conduite dans un véhicule en mouvement au sein d'un flux routier environnant et à en identifier les enjeux (section 3). Enfin, les apports et limites de cette recherche seront discutés du point de vue de la conception, mais aussi des outils et concepts théoriques mobilisés (section 4).

\section{Définir la reprise en main}

5 Après une revue de littérature (2.1), cette section introduit les théories que nous mobilisons (2.2 et 2.3 ) pour définir l'activité lors de la reprise en main par le conducteur (2.4). 


\subsection{Comment la reprise en main est-elle traitée dans la littérature ?}

La question de la reprise en main est fréquemment abordée comme pendant de l'automatisation des véhicules, elle-même déclinée en niveaux croissants décrits par la Society of Automotive Engineers (SAE, 2018). Ce document de référence pour la filière automobile distingue les paliers d'automatisation en fonction de la couverture par le système de la tâche de conduite (dite Dynamic Driving Task, DDT), en suivant la catégorisation proposée par Michon (Michon, 1985). Cette dernière décompose la tâche de conduite en trois types de processus hiérarchisés : (1) les processus stratégiques, qui concernent la planification de l'itinéraire et nécessitent un investissement cognitif important, (2) les processus tactiques, qui permettent l'anticipation des situations routières à gérer et la prise de décision des actions à venir lors des manœuvres, et (3) les processus opérationnels, qui concernent l'exécution des actions, souvent fondée sur des automatismes. En se basant sur ce modèle, la SAE définit les niveaux d'automatisation les plus bas, niveaux 1 et 2 , comme une automatisation partielle ou complète des processus opérationnels, le niveau 3 comme automatisation totale des processus opérationnels et tactiques, enfin les niveaux 4 et 5 comme stades d'automatisation augmentés par l'ajout d'une capacité du système à récupérer ses propres erreurs (DDT fallback: par exemple arrêter le véhicule quand le système est identifié défaillant) ${ }^{3}$.

7 Cette définition décrit bien le comportement du système technique : elle dit ce que le système prend en charge, et suppose, en creux, ce que le conducteur peut déléguer puis (re)prendre en main. Comme illustrée (pour le niveau 4) dans la Figure 1, elle invite à définir la «reprise en main » - et inversement le «détachement de la conduite » comme une transition entre différents niveaux d'automatisation (voir revue de littérature dans Lu et al., 2016).

Figure 1 : Définition de la notion de reprise en main pour le niveau 4 selon les niveaux SAE d'automatisation (simplifiés).

Figure1: Definition of take-over for level 4 according to SAE levels of automation (simplified)

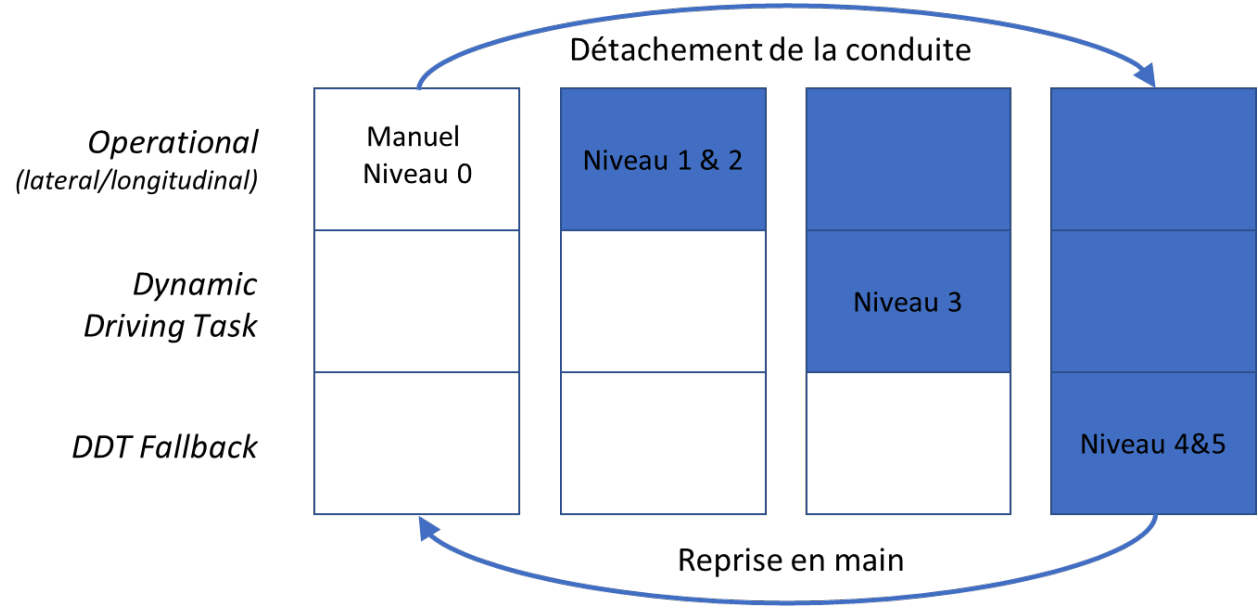

8 Ce modèle sert de base à la définition expérimentale des nombreuses études mesurant le temps nécessaire au conducteur pour effectuer une reprise en main, défini comme le délai entre la demande du système de reprendre la main et la désactivation effective de la fonction de conduite autonome, par un appui bouton par exemple (Dogan et al., 2017 ; 
Gold et al., 2013 ; Zhang et al., 2019). Dans cette lignée, la performance de conduite est étudiée au moment de la reprise par le conducteur, par exemple en termes de stabilité de trajectoire.

9 Un deuxième groupe de travaux aborde la reprise en main sous l'angle de l'évolution de la «conscience de la situation" du conducteur - ou Situation Awareness (SA). Il ne s'agit plus d'étudier la performance de la reprise en main en termes de temps de réaction ou de trajectoire, mais plutôt la performance cognitive du conducteur, envisagée sous l'angle de la complétude de sa perception de l'environnement (conscience d'un véhicule rapide arrivant par l'arrière, par exemple). Pour une partie de ces recherches, l'enjeu est d'évaluer la robustesse du système conducteur/véhicule face aux risques latents que le conducteur n'aurait pas anticipés (Gibson et al., 2016).

10 La Situation Awareness est classiquement définie comme « la perception des éléments de l'environnement dans un volume de temps et d'espace, la compréhension de leur signification et la projection de leur statut dans le futur» (Endsley, 1995b, notre traduction). Plus trivialement, il s'agit de "savoir ce qu'il se passe de façon à trouver ce qu'il faut faire ${ }^{4}$ (Adam, 1993). La SA est par conséquent conçue comme un état de connaissances et d'anticipations. Elle a également été étudiée comme un processus de construction de cette connaissance grâce à un monitoring régulier de son environnement par le conducteur, qui permet de suivre la position et la vitesse des véhicules alentour, ainsi que la progression des panneaux et embranchements. Lu et al. (2017) ont ainsi montré qu'il fallait observer l'environnement sur une durée de 7 à 12 secondes pour avoir une bonne idée des présences et positions respectives des véhicules environnants, et qu'une durée de 20 secondes devenait nécessaire pour avoir une idée précise de leurs vitesses respectives.

11 Le modèle qui s'est imposé comme référence pour une description de la Situation Awareness est celui proposé par Endsley (1995a, 1995b). C'est un modèle de prise de décision dynamique qui s'appuie sur trois processus successivement enchâssés. Le premier assure la perception de l'environnement. Il est englobé dans un processus de compréhension, lui-même englobé dans un processus d'anticipation des états futurs. Plusieurs méthodes ont par la suite été proposées pour mesurer l'exhaustivité de la perception des éléments de la scène de conduite par le conducteur, basées sur des questionnaires posés à un moment où la simulation de conduite est soudainement arrêtée en pleine action (par exemple, la méthode SAGAT) ou a posteriori après la reprise en main (voir la revue Gugerty, 2011).

12 Cette approche porte la vision d'un processus linéaire, très générique, qui ne décrit pas la particularité de la situation considérée. Chalandon (2007) questionne cette vision hors contexte et propose de préciser le rôle joué par l'environnement en mobilisant la notion d'affordance héritée de Gibson (Gibson, 1979). De façon schématique, la notion d'affordance permet de désigner les caractéristiques de l'environnement pertinentes pour l'humain ${ }^{5}$. Gibson a appliqué lui-même la notion d'affordance au champ automobile dans une étude où il a défini la notion de champ de déplacement sûr ("field of safe travel") comme ensemble des trajectoires favorables du véhicule considéré, en fonction de ses possibilités propres, des caractéristiques de l'infrastructure et des trajectoires à venir des autres objets mouvants (Gibson, 1938).

13 Chalandon mobilise également la notion de schème (Piaget, 1947) pour préciser les contenus "psychologiques» de la situation awareness, en basculant cette fois côté utilisateur. Les schèmes décrivent les principes d'action façonnés par l'acteur au cours 
de sa vie, "la structure ou l'organisation des actions telles qu'elles se transfèrent ou se généralisent lors de la répétition de cette action en des circonstances semblables ou analogues " (Piaget \& Inhelder, 1969). Ils formulent une possibilité d'action quand l'environnement est assimilé à un objet ou une situation connue. Quand aucun schème n'est disponible ou qu'il n'est pas tout à fait adapté, un schème accommodé aux nouvelles circonstances peut être construit dynamiquement (processus d'accommodation) et éventuellement approprié. C'est donc bien l'acteur qui, au cours de son histoire, construit les outils qui lui permettent d'interpréter et répondre à l'environnement. Dans cette perspective, la situation awareness comme une prémisse à l'action opère une assimilation/ accommodation de la situation rencontrée à une situation déjà connue à laquelle l'utilisateur sait réagir. Peu est dit en revanche sur les dynamiques de mises en œuvre de ces schémas d'action.

\subsection{La conduite comme boucles de couplage de pilotage et d'insertion dans le trafic}

14 Pour aborder la question pratique de reprise en main, nous proposons de considérer d'emblée, et non pas en sortie, l'interaction des utilisateurs du véhicule autonome avec leur environnement. Nous suggérons de partir d'une vision du système dans lequel interagit l'utilisateur, en considérant cette entité mixte (l'interaction) comme évolutive et centrée sur le conducteur: le véhicule et l'environnement de conduite doivent pouvoir s'y intégrer, s'en détacher au moins partiellement lors de la conduite autonome, et y revenir lors de la reprise en main. L'interaction est ainsi regardée comme ensemble de processus dont la structure se modifie avec le temps.

Les recherches théoriques rassemblées dans le paradigme de l'énaction (Maturana \& Varela, 1987) offrent alors un cadre privilégié pour accompagner cette perspective. La notion de couplage structurel revient précisément à définir l'interaction entre un humain et son environnement comme une interaction asymétrique, dont les potentialités sont portées par l'autonomie du "pôle » Humain, chargé d'une histoire et de dispositions qui orientent sa relation au monde. L'énaction décrit précisément le processus par lequel l'acteur se construit au cours de son histoire en s'appropriant ses interactions avec l'environnement (autopoïese) et en les inscrivant dans sa structure physique (ex.: musculation, connexions neuronales permises par la plasticité du cerveau). L'interaction est déterminée par l'acteur, plus que par l'environnement, précisément du fait de l'inscription dans sa structure physique de l'interaction passée : l'acteur s'approprie différents couplages passés et les nouveaux contextes qu'il rencontre réactivent, ré-énactent, ces mémoires. Ainsi, l'acteur construit et maintient un « filtre » de ce qui est significatif pour lui au vu de ce qu'il a fait précédemment et de ce à quoi il est prêt à réagir.

Tel que nous l'avons mentionné plus haut (cf. 2.1.), la pratique de conduite automobile est traditionnellement pensée, depuis plus de trente ans, à l'aide du modèle de Michon (1985), qui propose trois niveaux de description: opérationnel, tactique et stratégique. Cette décomposition a l'avantage de pointer la diversité temporelle des processus en jeu dans la conduite, mais elle présente la rigidité des modèles hiérarchiques et ne propose pas d'articulation structurelle entre ces niveaux. Une réinterprétation systémique de l'activité de conduite comme couplage acteur/environnement a été proposée par Haué (2005), en s'inspirant à la fois du paradigme de l'énaction, repris 
dans le programme de recherche Cours d'action, et du courant de cognition distribuée (Hutchins, 1995a ; Hutchins, 1995b). Selon cette proposition, le système de l'activité de conduite se déploie dans la situation concrète en boucles de couplage en y intégrant les éléments de l'environnement pertinents (éléments du véhicule, infrastructures, règles de conduite et de circulation, etc.).

Une mise en correspondance de cette proposition, qualifiée de modèle systémique, avec les niveaux du modèle de Michon est proposée dans la Figure 2.

Figure 2 : Mise en correspondance du modèle de Michon et d'une réinterprétation systémique (Haué, 2005).

Figure2: Correspondences between Michon's model and a systemic formulation (Haué, 2005)

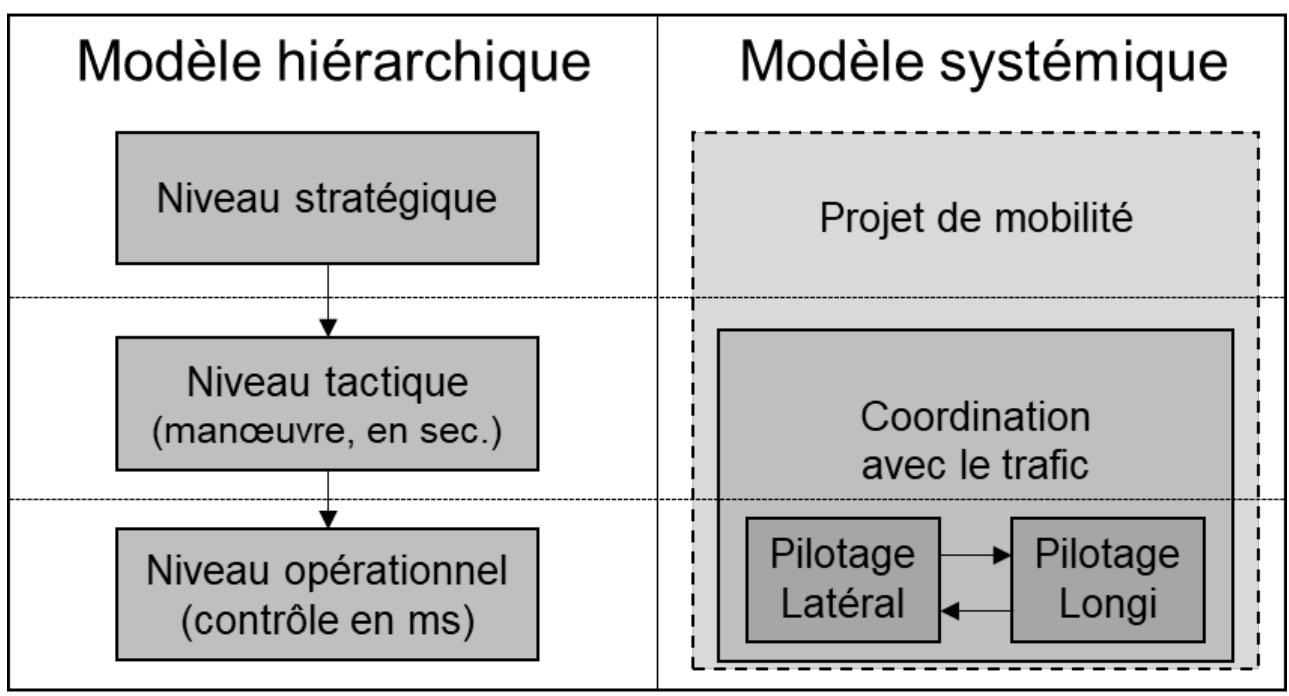

Le modèle systémique propose de redéfinir les boucles de contrôle du niveau opérationnel en boucle de pilotage. La boucle dite de pilotage latéral, visant à « maintenir la trajectoire ", relie la façon dont le conducteur projette la trajectoire de son véhicule sur la route et les actions correctrices qu'il met en place (par ex., en tournant légèrement le volant), pour ajuster en temps réel les écarts entre la trajectoire réelle et celle souhaitée. La boucle de pilotage longitudinal, quant à elle, consiste en la gestion de la vitesse selon la distance avec le véhicule de devant, ou selon la vitesse réglementaire ou perçue comme sûre. Ces deux boucles peuvent constituer des soussystèmes relativement autonomes, mais s'intègrent dans un système plus vaste par exemple dès que la vitesse dans un virage peut avoir une incidence sur la trajectoire.

Les boucles du couplage associées au niveau tactique constituent un sous-système qui concerne la capacité du conducteur à maintenir une certaine coordination avec le trafic. Il s'agit alors de maintenir une perception du trafic environnant, des infrastructures et changements de route afin d'évaluer comment le véhicule est inséré dans le flux environnant et de s'y adapter en temps réel. L'anticipation d'éventuelles perturbations de ce flux amène à porter intérêt aux opportunités d'adaptation (par exemple, un espace dans une voie adjacente) et d'anticiper les manœuvres pour les réaliser (par exemple, un changement de voie). Lors de l'anticipation ou de la réalisation d'une manœuvre, les sous-systèmes de pilotage sont étroitement intégrés dans le système de coordination avec le trafic.

20 La différence essentielle entre les deux modélisations est que le modèle systémique prend en compte l'historicité de l'acteur et le rôle de la situation. L'activité de conduite 
y prend place au sein d'un projet de mobilité global porté de l'acteur au sein duquel les boucles de coordination avec le trafic, de pilotage latéral et longitudinal se déploient en constituant des sous-systèmes relativement autonomes, ou en interagissant entre eux selon les circonstances de la situation.

\subsection{Penser la dynamique d'engagement avec le Cours d'Action} définir leur dynamique de mise en œuvre. Le signe hexadique (Theureau, 2006) offre précisément un outil pour décrire de la manière la moins réductrice possible cette dynamique de couplage. Il est ici appliqué à la reprise en main, telle qu'elle est vécue par l'acteur en termes de formes d'engagement.

hexadique, issu de l'approche Cours d'Action, croise la théorie du couplage structurel évoquée en 2.2 avec les apports de la sémiologie de Peirce. Il traduit l'hypothèse de l'«activité signe » comme quoi le processus d'énaction qu'est l'activité s'accompagne d'une émergence continuelle de sens. Cette signification est à la fois ce qui dirige l'activité dans son déroulement et ce qui apparaît à l'acteur' ${ }^{6}$.

Pour aller décrire le contenu de ce processus, Theureau reprend alors de Peirce (1978) ses trois catégories de la dynamique d'émergence d'un système (Figure 3 en haut à gauche) :

1. La catégorie du Possible, qui décrit l'état, la posture, la qualité de présence, le potentiel d'interaction avant contact au monde qui circonscrit, positivement comme négativement, le champ des possibles pour le système, les « envisageables ».

2. La catégorie de l'Actuel, qui est celle de la rencontre avec le monde, avec le réel envisagé comme altérité. Elle contient en particulier ce qui fait choc, l'élément de l'environnement retenu comme pertinent par rapport au possible.

3. La catégorie du Virtuel, qui décrit la loi réalisée pour résoudre la transformation initiée par le choc, acquise lors d'expériences passées ou « inventée » dans une situation nouvelle.

La figure en bas à gauche montre l'application des catégories sémiotiques peirciennes à la rencontre entre la structure de l'acteur (son corps, son système nerveux) et l'environnement. Les catégories de Possible/Actuel/Virtuel rendent bien compte de l'hypothèse d'asymétrie de couplage portée par l'énaction : l'acteur 1) se construit luimême au cours de son histoire (Virtuel/appropriation) et 2) sélectionne par son état de préparation ce qui est significatif pour lui, dans une interaction asymétrique (Possible ; état de préparation $\left.{ }^{7}\right)$. 
Figure 3 : Construction du signe hexadique offrant une grille de lecture à l'émergence de l'expérience.

Figure3: Construction of the "hexadic sign" provides an analysis grid for the emergence of experience
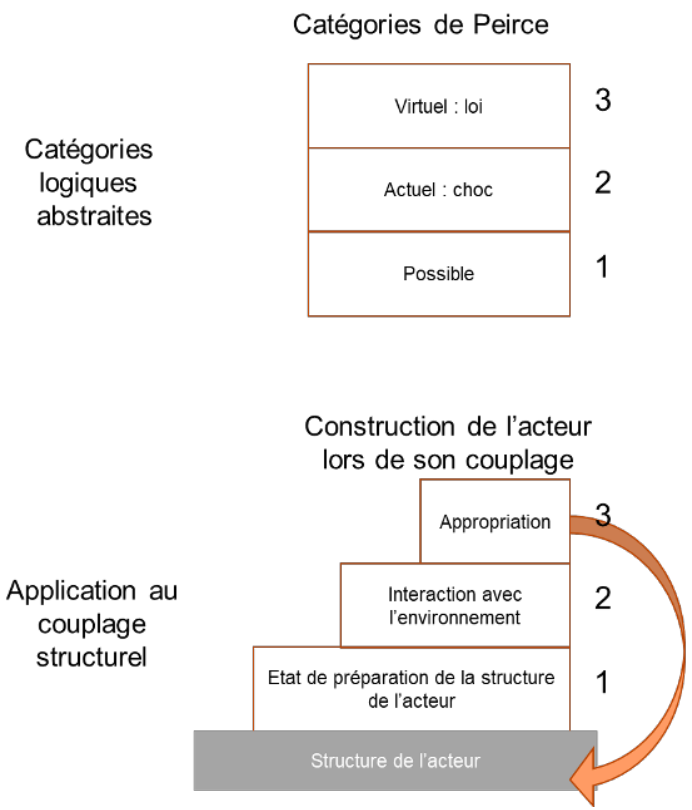

$\left(\right.$ Peirce $\left.^{2}\right)$ repris par Theureau

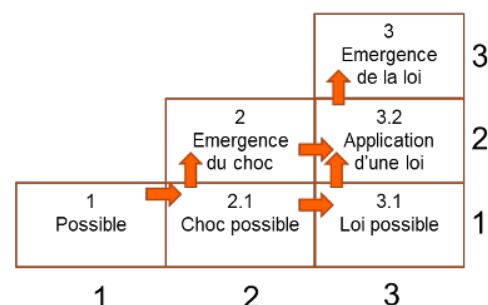

Signe hexadique : Emergence du sens au cours du couplage

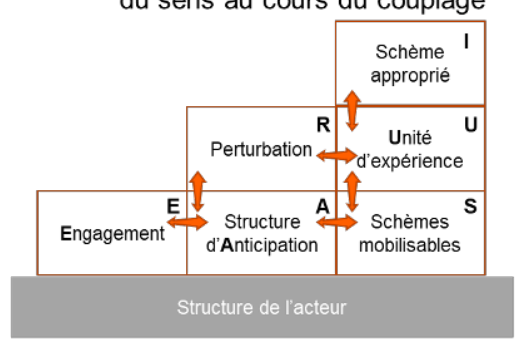

Theureau repasse alors par l'inspiration logique et croise ces catégories pour décrire la construction de signification dans la rencontre au monde (choc au monde), à partir de la réactivation des interactions passées (Figure en haut à droite). Le choc au monde se décompose en chocs possibles (2.1, ce qui est « attendu»), dont un s'actualise (2). De la même manière, la manifestation d'habitudes, façons de faire, savoirs, types se comprend comme une loi actualisée (3.2.), sélectionnée sur un fond d'habitudes, façons de faire... mobilisables à ce moment-là, autrement dit sur fond de lois possibles (3.1).

Le signe hexadique (figure en bas à droite) fournit en définitive un outil opérationnel pour l'analyse. Il permet de décrire comment, dans un moment d'activité, l'engagement (E) oriente l'activité vers différents objets, i.e. différents champs de possibles, en l'occurrence, pour nous, champs relatifs à la vie à bord ou à la conduite. Chacun de ces objets vient ouvrir un espace dans la structure d'anticipation (A), où se créent des attentes sur la continuité et l'évolution de l'objet suivi, qu'il s'agisse de pensées ou souvenirs, de buts fixés ou simplement du suivi du déroulement d'un fil d'événement. Par exemple, lors du pilotage de son véhicule, le conducteur suit l'évolution de sa trajectoire par rapport à une trajectoire anticipée. De même, il maintient une attente sur sa vitesse ou la distance avec le véhicule le précédant.

Le Référentiel (S) est quant à lui constitué de types, relations entre types, et principes d'interprétation. Dans la suite de l'article, nous opérons l'approximation d'assimiler ces notions à celle de schèmes mobilisables, qui correspond à l'ensemble des principes d'action ou d'interprétations sélectionnés comme pertinents par rapport à la structure d'anticipation. Les attentes et anticipations maintenues dans les champs de possibles ouverts dans l'engagement "accrochent " et maintiennent mobilisables les schèmes acquis qui permettraient d'interpréter ou de réagir à ces attentes. L'ensemble des schèmes maintenus mobilisables, tant d'action, d'interprétation, de jugement, qu'émotionnel..., fournit le référentiel pragmatique de la situation, l'horizon des actions possibles. 
L'état de préparation orienté par l'engagement circonscrit tout d'abord par la structure d'anticipation, ce qui est susceptible de faire signe à l'acteur dans la situation. Ensuite, quand la perturbation $(\mathrm{R})$ actualise cette anticipation, le référentiel propose des schèmes mobilisables dont éventuellement celui pertinent pour la résoudre dans l'unité d'expérience (U) qui apparaît alors à l'acteur. L'interprétant (I) manifeste finalement la construction de sens et les connaissances éventuellement nouvelles qui pourront être appropriées.

En synthèse, le signe hexadique fournit une grille d'analyse fine pour étudier comment, dans un processus de création de sens, l'engagement découlant des interactions passées évolue, maintient, lâche ou active des états de préparation de l'acteur qui cadrent l'interaction avec l'environnement. Il permet de décrire comment le couplage de conduite se ré-établit lors de la reprise en main. Par la structure d'anticipation (EAS) liée au système de coordination avec le trafic, il caractérise la connaissance de la scène de conduite qui se construit à cette occasion.

\subsection{Définition du réengagement à la conduite}

Les développements précédents nous permettent de redéfinir la reprise en main et le détachement à la conduite dans les termes dérivés du signe hexadique de désengagement et réengagement au monde de la conduite.

31 D'un point de vue conceptuel, l'engagement désigne la première composante du signe hexadique, i.e. l'état d'équilibration, la dynamique qui ouvre et maintient des espaces de couplage. Sur le plan opérationnel d'une description des activités en cours, l' engagement - dans la conduite ou dans une autre activité comme la vie à bord - désigne l'ouverture d'un champ de possibles qui amène l'apparition 1) d'attentes associées dans la structure d'anticipation (dans A) et 2) de schèmes mobilisables (dans S). L'engagement dans la conduite peut être orienté vers les boucles de pilotage et/ou de coordination avec le trafic (cf. section 2.2).

Réciproquement, le désengagement de la conduite (ou d'une autre activité) désigne le processus par lequel la structure attentionnelle se détourne du monde de cette activité : le champ des possibles dirigé vers l'activité initiale de conduite n'est plus maintenu dans la structure d'anticipation et se dissipe, pouvant aller jusqu'à disparaître en totalité. C'est notamment ce qui peut se produire lors d'une activité annexe - dite tâche secondaire - lorsque celle-ci absorbe le conducteur dans un monde qui lui est propre, et disjoint du monde automobile.

33 Enfin, le réengagement dans la conduite (ou d'une autre activité) désigne une réouverture des possibles qui s'étaient partiellement ou totalement dissipés. La réactivation de l'engagement de l'acteur devient alors a priori d'autant plus facile et rapide qu'il restait rémanent.

Nous montrons en section suivante comment ces notions nous ont permis de mener une analyse fine d'exemples de reprise en main et de mettre en évidence la coexistence et l'évolution de différentes formes d'engagement. 


\section{Données empiriques : une étude menée sur simulateur}

L'étude présentée dans cette section, réalisée en 2015 (Beun, 2015), a été initiatrice d'un programme de recherche empirique trisannuel (Le Bellu \& Haué, in prep.). Son objectif était exploratoire. Il s'agissait spécifiquement d'obtenir des premières mesures de temps de reprise en main en fonction de différentes localisations d'un écran de visualisation de contenus vidéo, consultables lors des phases de conduite autonome, et de recueillir de premières observations qualitatives sur les processus de désengagement et de réengagement dans la conduite. C'est sur les résultats des observations qualitatives que nous nous attardons dans cet article. Le protocole d'expérimentation de cette étude est d'abord présenté (3.1.). Nous montrons ensuite avec un exemple d'analyse détaillé de l'un des participants comment la théorie du Cours d'Action présentée plus haut permet l'analyse des processus de désengagement/ réengagement (3.2.).

\subsection{Dispositif d'expérimentation et protocole}

L'étude s'est déroulée sur un simulateur léger nommé S2EI (Simulateur d'Étude Ergonomique d'IHM, Figure 4), doté d'une IHM représentant un tableau de bord minimal et permettant de visionner un contenu vidéo. Ce simulateur se compose d'un poste de conduite (siège, volant), d'un grand téléviseur montrant la scène de la simulation de conduite et d'écrans supplémentaires pour piloter le véhicule et visionner le film à différents endroits (écran haut, écran bas, tablette manuelle).

Dix-sept participants volontaires, onze hommes et six femmes, âgés de 22 à 49 ans, ont été recrutés parmi les 12000 salariés du site. Aucun d'entre eux n'était lié au projet.

Figure 4 : Simulateur S2EI (Simulateur d'Étude Ergonomique d'IHM). Figure 4: S2EI simulator

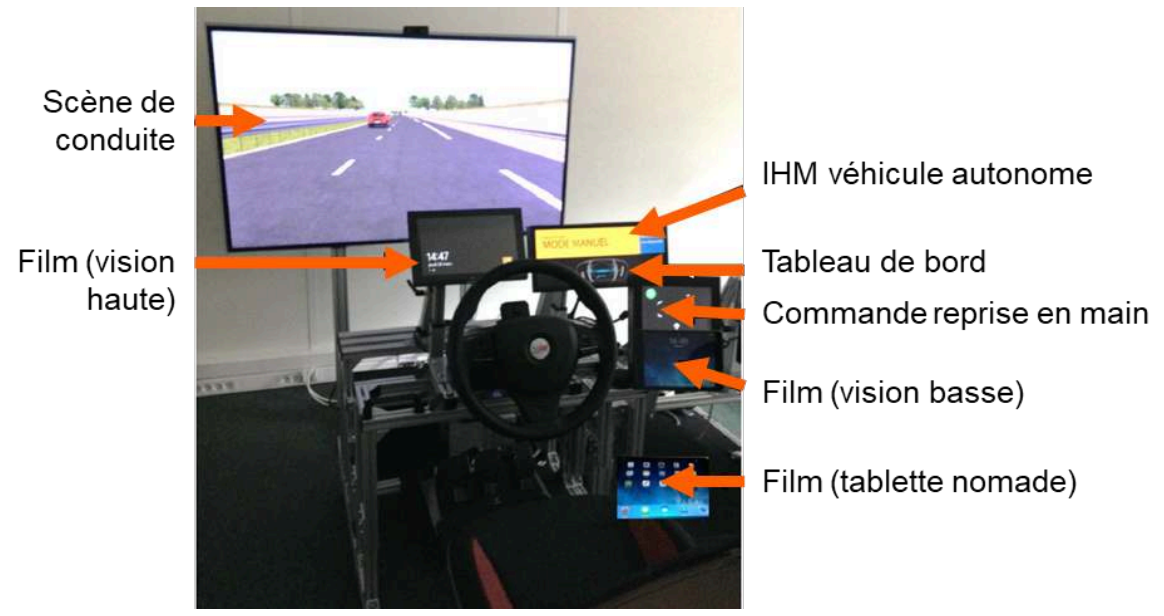

Le simulateur était équipé de deux caméras orientées de façon à recueillir le son, les enregistrements vidéo des visages/postures/pieds des participants, ainsi que la scène de conduite. Les participants étaient de surcroît équipés d'un eye-tracker, fournissant un enregistrement de leur champ de vision avec la position de leur regard. 

chacun (Figure 5), pour un temps total sur simulateur d'environ 40 minutes ${ }^{8}$.

Figure 5 : Phases de l'expérimentation de conduite sur S2EI.

Figure 5: Phases of the S2EI driving experiment

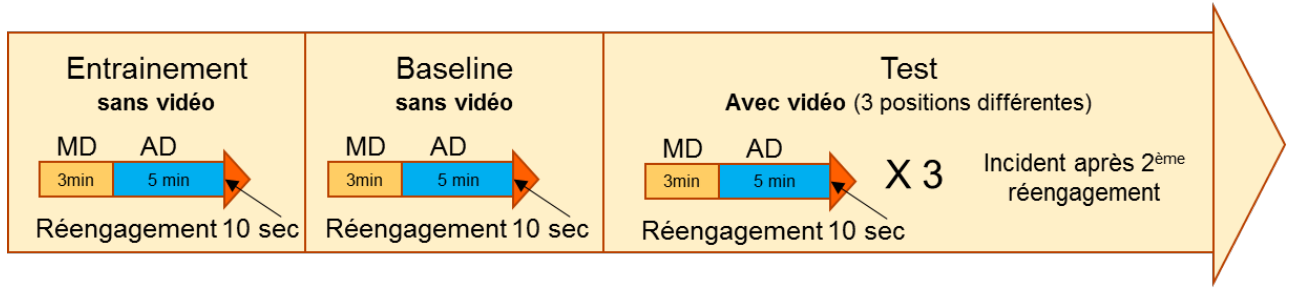

Chacun des scénarii incluait une première phase de 3 minutes en conduite manuelle (Manual Driving, ou MD) à l'issue de laquelle l'IHM indiquait que le mode autonome (Autonomous Driving, ou AD) était disponible, activable par la commande située sur l'écran à droite. Le participant basculait en conduite autonome au moment de son choix, puis, après 5 minutes, l'IHM émettait une demande visuelle et sonore de reprendre la main en 10 secondes, déroulées par un compte à rebours.

41 Le premier scénario était une session d'entrainement et de découverte à la fois du simulateur et $\mathrm{du}$ fonctionnement du système de conduite autonome simulé, limitant ainsi les biais inhérents à la découverte d'un système. Le second scénario (baseline), identique au premier, servait de référence pour comparaison avec les scénarii avec vidéo.

Les trois scénarii de test qui suivaient incluaient une tâche de vie à bord durant la période de conduite autonome, qui consistait à visionner un contenu vidéo, tâche considérée comme a priori motivante et sollicitante afin de maximiser le désengagement de la conduite et la bascule vers l'engagement dans la vie à bord. Le contenu vidéo, "La vie rêvée de Walter Mitty", avait été choisi à cet effet. L'emplacement physique de l'écran de visionnage du contenu vidéo variait selon le scénario. Le contenu vidéo s'arrêtait automatiquement lors de la demande de reprise en main, sauf sur la tablette.

Dans l'un de ces trois scénarii, un événement potentiellement dangereux, à savoir un véhicule dépassant par la gauche puis se rabattant et freinant brutalement devant le participant, était scénarisé moins de 6 secondes après le retour à la conduite manuelle. Cet événement avait pour but de tester la réactivité du conducteur.

À l'issue de la passation sur simulateur, un entretien semi-directif était réalisé afin de recueillir des évaluations des IHM ainsi que le ressenti global de l'expérimentation. Deux participants de l'échantillon, que nous nommerons P1 et P2 dans la suite du texte, ont été sélectionnés pour une analyse plus approfondie, car ils montraient des processus de réengagement contrastés. Un entretien d'autoconfrontation complémentaire a été mené avec chacun d'eux afin de recueillir la verbalisation détaillée de leur vécu sur la base de l'enregistrement vidéo de leur réengagement lors du scénario de test incluant l'événement potentiellement dangereux. 


\subsection{L'analyse détaillée d'un cas de désengagement et de réengagement}

La moitié des dix-sept participants n'a pas réussi à éviter l'accident avec le véhicule se rabattant en freinant brutalement, ce qui peut s'expliquer en partie par le besoin de réengagement après la tâche de vie à bord, mais aussi par les limites techniques du simulateur S2EI (position du bouton, sensations limitées de la trajectoire et de la vitesse qui gênent la stabilisation, ou encore absence de rétroviseurs n'ayant pas incité à regarder autour). L'analyse des données montre également que les accidents ont été majoritairement subis par les plus jeunes des participants (moins de 34 ans), dont l'immersion a été qualitativement observée comme étant beaucoup plus intense que pour leurs aînés lors du visionnage du contenu vidéo.

Le premier des participants choisis pour l'analyse approfondie (P1) a évité la collision sans difficulté, en ayant constamment gardé une partie de son attention sur la conduite pendant le temps de conduite autonome, et ce, malgré le visionnage du contenu vidéo. Le second, P2, ne l'a quant à lui évité que de justesse, après un désengagement fort de la conduite. L'analyse détaillée de l'épisode très critique de conduite de P2 est présentée ci-après afin de comprendre ses processus de désengagement et de réengagement.

La Figure 6 montre la reconstitution du déroulé de ce moment d'activité à l'aide des catégories du signe hexadique. La partie supérieure de la figure synthétise la chronologie, avec mise en correspondance d'une description de l'environnement, du mode de conduite activé, et de l'analyse du cours d'action du participant. La partie inférieure de la figure propose un découpage de l'activité en Unités d'expérience du cours d'action et une reconstitution de l'état de préparation de la participante (Engagement, Structure d'anticipation, Référentiel) grâce à l'analyse conjointe des données sur l'environnement, des enregistrements des comportements de la participante, et, lors de l'autoconfrontation, de l'explicitation par la participante de son expérience vécue. 
Figure 6 : Analyse qualitative d'une phase de réengagement basée sur l'étude de l'activité de P2 dans l'étude S2EI.

Figure 6: Qualitative analysis of a reengagement phase based on the activity study of $P 2$ in the S2El study

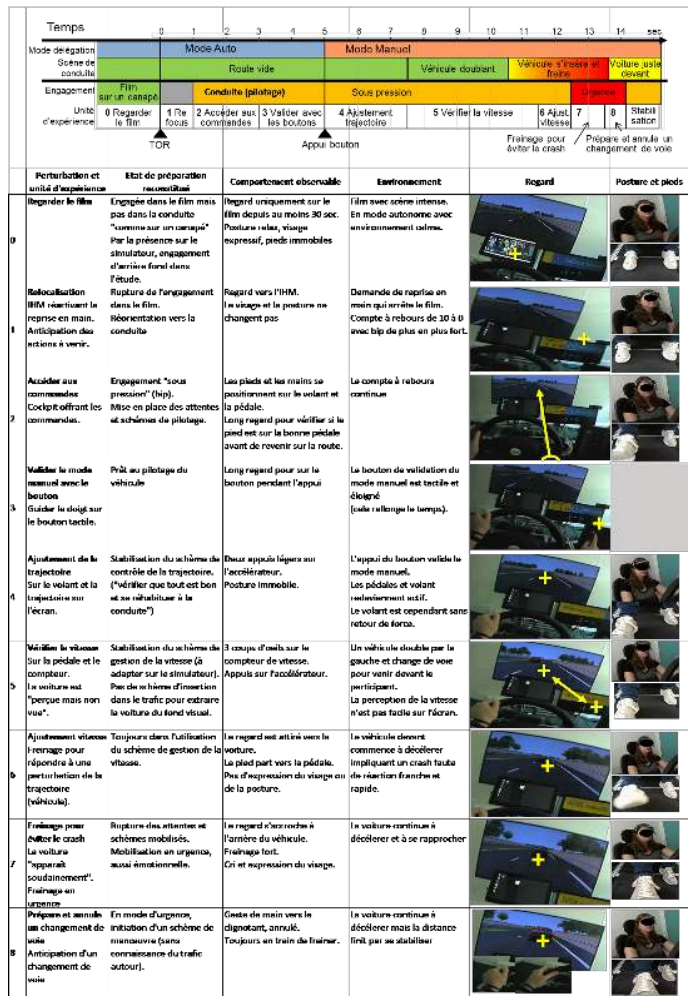

Cette analyse illustre une situation de désengagement complet de la conduite, manifestée par la fixation du regard depuis au moins 30 secondes sur le contenu vidéo et par l'expérience rapportée par la conductrice lors de l'entretien où elle se déclare " plus du tout en train de conduire ", " comme sur un canapé » (Unité d'Expérience 0 dans la Figure 6). Ce désengagement complet de l'activité de conduite n'a pas été manifesté par certains participants, notamment P1, dont l'activité oculaire était caractérisée par des fixations régulières sur la scène de conduite, et qui a décrit a posteriori avoir " résisté " pour ne pas rentrer dans le contenu vidéo.

Juste après le signal sonore demandant la reprise en main, une période de latence d'une seconde a été observée. La posture et l'expression de la conductrice sont restées identiques à celles du moment précédent. Celle-ci a indiqué lors de l'entretien être en train de se préparer aux actions à venir. Pour elle, le signal sonore aide, même si, plus tard, il deviendra stressant. Nous interprétons cette période comme une phase où, après un effondrement de son engagement dans le film, la participante réoriente son attention vers la reprise en main. Nous la renommerons par conséquent refocalisation (UE1).

50 La reprise en main se manifeste d'abord par une reprise d'une posture de conduite (UE 2 : accéder aux commandes) suivie par la validation du mode manuel (UE 3 : valider le mode manuel avec le bouton). La conductrice se focalise alors sur ses réactions de pilotage, appelées par les commandes du cockpit et le besoin immédiat de contrôle du véhicule (UE 4 : ajustement de la trajectoire). La perception très tardive du véhicule freinant devant est significative de l'absence de mise en place de la boucle de coordination dans le trafic décrite dans la section 2.2 (UE 5 : vérifier la vitesse). Tout se passe comme si la 
conductrice n'était pas encore présente à la situation plus large qui l'environne. Le véhicule tiers à ce moment-là est décrit dans l'entretien comme "perçu, mais pas vu ", interprété comme une perception dans l'arrière fond de l'environnement, i.e. non filtré comme pertinent à ce moment-là par l'acteur, ne laissant donc pas de place pour d'éventuels schèmes d'action.

51 La réaction à la perception du véhicule devenu très proche se fait finalement par le biais d'un freinage (UE 6 : ajustement vitesse). Ce freinage est très rapidement suivi d'une réaction beaucoup plus forte manifestée par un cri de la part de la participante et d'un freinage d'urgence (UE 7 : freinage pour éviter le crash). Le véhicule tiers est décrit lors de l'entretien post-hoc comme "apparaissant subitement ", à une distance déclenchant une réaction d'urgence et une mobilisation intense du schème associé. Un début de changement de voie est finalement initié, sans avoir contrôlé la présence d'un véhicule sur le côté (UE 8 : prépare et annule un changement de voie).

La chronique montre finalement un état de désengagement complet de la conduite et l'effort pour s'y réengager en refocalisant l'attention dans la reprise de possession du véhicule et de l'environnement. L'effort à fournir dans ce temps très limité amène à reconstruire le couplage le plus immédiatement proposé par l'environnement (la posture et les commandes) et le plus urgent: le pilotage du véhicule. À l'inverse, la reconstitution du système de coordination avec le trafic, moins directement sollicité par l'environnement et demandant un temps plus long (de l'ordre de 10 à 20 secondes tel qu'évalué dans l'étude citée dans la section 2.1) est négligé. Il en résulte le comportement de reprendre la conduite sans avoir de perception de l'environnement de conduite autour de soi.

Cet exemple présente l'intérêt d'illustrer la temporalité progressive d'un réengagement à la conduite suite à un désengagement prononcé - en raison ici d'un engagement concurrent dans une activité tierce fortement sollicitante. Il montre un réengagement partiel à la conduite se manifestant par la reconstruction d'un couplage de pilotage adéquat, mais aussi par le manque de reconstruction du couplage de coordination avec le trafic. Pour cette utilisatrice novice, le réengagement apparaît comme un processus dirigé par sa considération sur les étapes à réaliser (effectuée lors de cette seconde où elle se prépare aux actions à venir en UE1).

\section{Discussion}

\subsection{Engagement, environnement et activité}

54 Nous proposons en discussion de nous fonder sur l'analyse de la reprise en main de la participante 2 (P2) pour discuter des contraintes et ressources intervenant dans les changements de dynamique du couplage. Leur articulation est ici proposée dans les termes du Cours d'Action, notamment à partir des catégories E-A-S.

L'environnement apparaît dans le cas analysé comme à la fois un ancrage et une source de déstabilisation de l'activité en cours. Lors de la demande de reprise en main, la coupure du contenu vidéo supprime l'ancrage sur lequel reposait l'activité de vie à bord et fait vaciller l'engagement de l'acteur. Il n'y a plus le flux d'image pour lequel étaient maintenus mobilisables les schèmes interprétatifs. Le compte à rebours visuel et le signal sonore répétitif, spécifiquement conçus afin d'interrompre l'activité en cours, fonctionnent effectivement comme tel: la participante rapporte qu'ils induisent un 
sentiment d'urgence et incitent à reprendre la main. Dans ce cas précis, l'environnement s'impose à l'acteur qui, plongé dans la vie à bord, n'était pas dans l'anticipation de cet événement. C'est la disparition des stimulations attendues de la part du film et l'alerte intrusive, dans le $\mathrm{R}$ du Signe 1 représenté Figure 7, qui déstabilisent et viennent bousculer l'état de préparation EAS en cours. Le fait marquant, au vu des analyses traditionnellement effectuées dans le cadre du Cours d'action, est que l'élément faisant choc (le R) sort ici du champ d'attentes de l'acteur ; il vient s'y imposer ou y imposer son absence avec pour conséquence d'effondrer la structure d'anticipation en vigueur. Ce point n'est pas trivial et offre un nouvel éclairage puisque dans notre perspective théorique l'émergence d'un sens est comprise comme actualisation sur fond de possibles interprétatifs.

Pour lors, nous observons que l'engagement de l'acteur s'étant écroulé, l'état de préparation doit se reconstruire en l'occurrence vers la conduite pour que le couplage se poursuive.

57 Même plongée dans le film, la participante P2 n'est pas totalement coupée de la route. La position sur le siège du conducteur dans un véhicule en mouvement représente déjà un engagement corporel et situationnel prêt à la ramener vers la conduite. P2 rapporte faire l'expérience d'une seconde où, sans mouvement apparent, elle "prépare ses actions à venir" (UE1 de la Figure 6). Vermersch (1994) offre des outils pour une description des processus en jeu lors de cette seconde : il identifie dans le processus d'assimilation/accommodation piagétien certaines circonstances susceptibles de résister à l'intelligence sensori-motrice d'un acteur (celle qui mobilise ses schèmes d'action). Cette rupture amène alors l'acteur à se focaliser sur des signifiants internes. Ces signifiants internes peuvent à leur tour servir de signe pour faire appel par association à d'autres expériences passées (Vygotsky, 1978). Ainsi, P2 a pu laisser remonter le souvenir symbolique ou sensoriel des quelques manœuvres de reprise en main vécues un peu plus tôt lors des précédents scénarii, des consignes d'information orale données par les expérimentateurs ou plus simplement de ses habitudes de conduite.

Dans cette étape, la réorganisation de l'état de préparation de l'acteur peut s'appuyer également sur des ressources de l'environnement perceptible. Le tableau de bord du cockpit de véhicule autonome est notamment porteur d'un ensemble d'informations (ex.: vitesse, mode de conduite, trajectoire du véhicule...), qui contribue à une distribution cognitive comme montré pour le cockpit d'avion par Hutchins (1995b). L'environnement physique offert par le cockpit constitue une forme de mémoire en inscrivant également dans l'objet une forme adaptée au geste de sa manipulation (Leroi-Gourhan, 2014). Par exemple, la forme et la localisation des commandes invitent à leur manipulation, et le bouton de reprise en main à son actionnement. Ces mémoires de l'environnement sont conçues pour offrir des représentamen supportant un type d'action visé dans la situation d'utilisation.

Dans cette seconde de réajustement de l'engagement, P2 semble finalement intégrer des éléments dans son état de préparation par la perception sensible et l'évocation de souvenirs (dans R) qui viennent enrichir et préciser la structuration de son anticipation. En plus de convoquer des expériences-types, les Unités d'Expérience semblent intégrer une série d'actions projetées (mais non réalisées) qui vient réveiller des schèmes mobilisables (signe 2 présenté Figure 7). Ces mécanismes de construction de l'engagement nous semblent préciser comment une unité d'expérience $U$ par cette 
phase de projection non réalisée transforme l'état de préparation EAS de l'acteur pour s'adapter en changeant la définition de la situation en cours. À nouveau, ce processus n'est pas trivial dans la perspective Cours d'Action où le signe hexadique est normalement tourné vers une action réalisée. Il apparaît ici que des cycles «à vide » peuvent se manifester pour consolider un état de préparation, avant le passage à l'action.

Quand l'état de préparation E-A-S est suffisamment stabilisé, il permet le passage à l'action (signe 3 de la Figure 7). Ce dernier se manifeste par l'implication du corps dans la situation, à l'instar de P2 qui après la phase de projection, se met en mouvement dans l'UE2 et les suivantes. L'activité de P2, précédemment dans la projection, se met alors à passer par les commandes du véhicule pour faire corps et percevoir directement à travers lui (Merleau-Ponty, 1945). L'implication de l'acteur dans le pilotage ancre alors l'état de préparation par ce couplage effectif avec l'environnement.

61 Finalement, ces différents mécanismes constituent toute une série de boucles de rétroactions locales sur l'état de préparation par ajustement de la signification projetée qui réoriente le couplage vers la conduite. Par contre, s'étant focalisée lors de son réengagement sur les boucles de pilotage et la trajectoire de son véhicule, notre conductrice filtre et "perçoit sans voir» le véhicule dont le rapprochement va la projeter brutalement et tardivement dans une boucle de coordination avec le trafic. Idéalement, tel que nous le discutons en section suivante, il serait souhaitable que la reconstruction de cette boucle de coordination se fasse plus en amont afin de rendre plus aisé et sécurisé le retour à la conduite manuelle.

Figure 7 : Signes hexadiques des trois étapes de réengagement. Figure 7: Hexadic signs of the three-step reengagement process

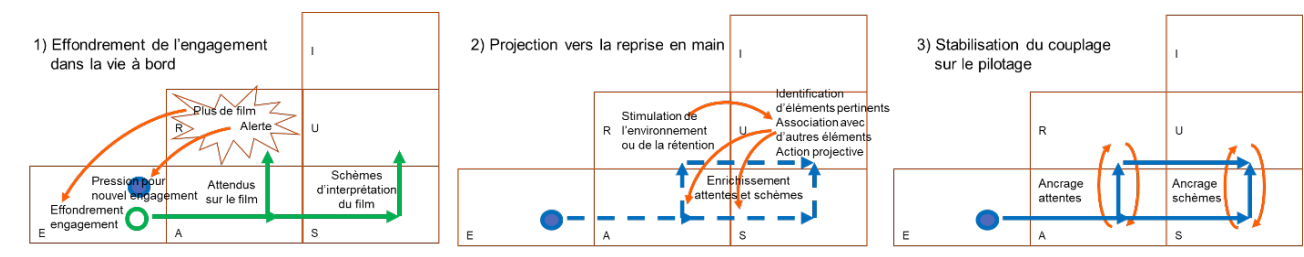

Cette approche située permet de reformuler la notion de Situation Awareness. Le modèle d'Endsley pose un processus linéaire de perception/compréhension/projection, qui présente l'avantage de lier perceptions à attentes, mais ne dit rien sur la mise en place de ce processus. Il ne dit rien non plus de la matière dont est constituée cette SA. Inspirés par la cognition située, Banks, Stanton et Harvey (2014) avaient ajouté un processus de feedback à cette séquence, sous la forme d'une phase d'anticipation. Ici, c'est toute une panoplie de boucles de stabilisation de la signification pour l'acteur qui s'est révélée par les rétroactions de l'activité sur l'état de préparation (EAS) pour s'adapter au changement de situation. Et c'est cet état de préparation qui nous semble constituer la Situation Awareness. Plus qu'un processus linéaire, cet état de préparation/SA peut davantage être vu comme un ensemble d'espaces, de fenêtres attentionnelles (Vermersch, 2002), maintenus et mis à jour en permanence. Ils s'ouvrent quand une action est entreprise ou quand une action précédemment ouverte, mais non terminée, maintenue comme une préoccupation par l'acteur, se réactualise lors d'un événement pertinent (Salembier, Theureau, Zouinar, \& Vermersch, 2001). Cette focalisation de l'attention est pondérée par la force de l'engagement. Cet espace est ancré par endroits aux éléments matériels de l'environnement, par le suivi des 
attentes et la mise en œuvre des schèmes. Il est également enrichi par les médiations symboliques ou sensorielles évoquant des éléments issus de la rétention de moments passés. Dans ce paysage d'attentes et d'opportunités d'actions, la dynamique de l'attention sera d'autant plus attirée à ces différents endroits que l'engagement y est fort.

La séquence de $\mathrm{P} 2$ a montré un désengagement fort de la conduite pour se plonger dans le film, demandant ensuite un effort important de réengagement à la conduite. Elle s'est focalisée sur les commandes du véhicule, amenant à se focaliser sur le pilotage.

P1 quant à lui avait résisté à l'attrait du film. Maintenir un engagement, bien que partiel, à la fois dans le film et à la conduite plus particulièrement sur le trafic, avait alors facilité la reprise en main. Nous généralisons dans la dernière section les processus d'évolution de cet espace d'engagement pour en tirer des recommandations pour la conception.

\subsection{Vers la conception : construction de l'« entonnoir de réengagement »}

Les exemples de P1 et P2 illustrent bien la variation rencontrée entre les autres participants de l'étude, par ailleurs retrouvée dans les études menées ultérieurement sur la conduite autonome (Le Bellu \& Haué, in prep.). La distinction entre les désengagements forts et limités d'un côté et celle entre le réengagement partiel (pilotage seulement) ou complet (pilotage et coordination) de l'autre invite à généraliser une typologie des processus de réengagement, proposée Figure 8.

Figure 8 : Typologie des processus de réengagement (boucle de Pilotage et de Coordination avec le trafic décrits section 2.2.).

Figure 8: A typology of reengagement processes

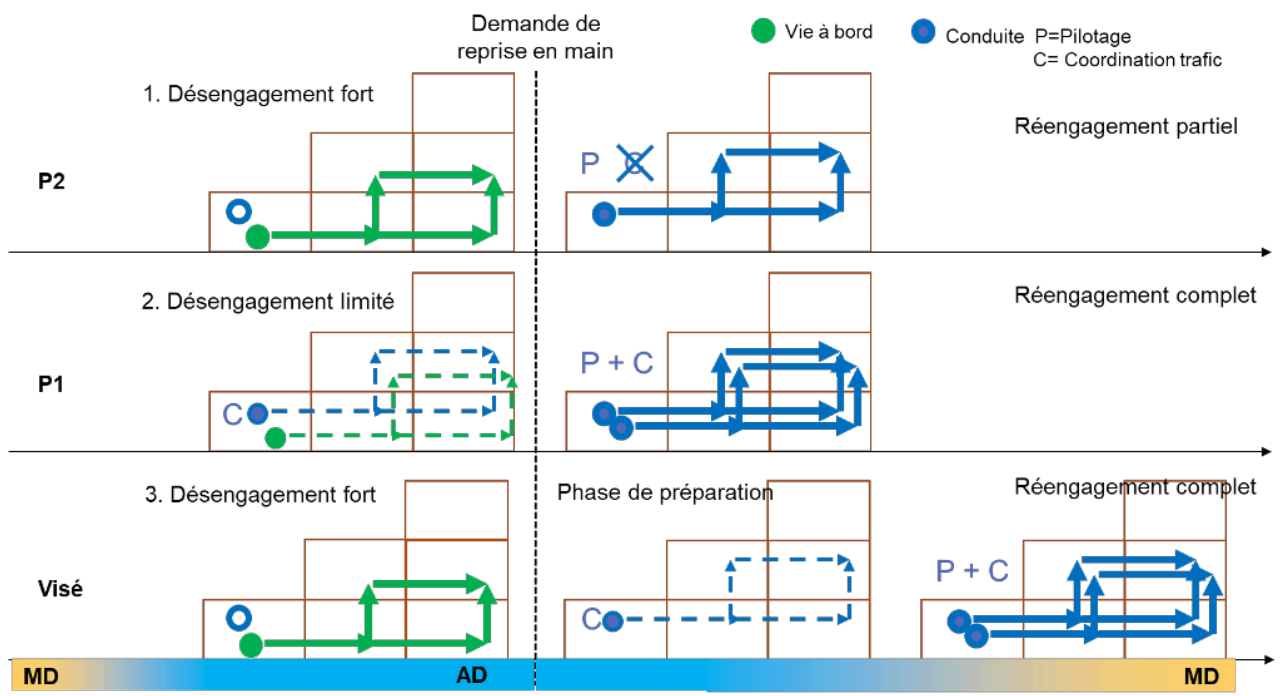

Le premier processus de réengagement partiel, faisant suite à un désengagement fort, correspond au cas analysé (P2). Le conducteur, très engagé dans la vie à bord, subit un réengagement « sous pression ». L'accès aux commandes est privilégié, comme détaillé dans la Figure 7. Le conducteur reprend la main sans avoir reconstruit une image de la situation en cours, et donc sans l'anticipation nécessaire en cas de changement de la 
scène routière tel qu'un événement dangereux à éviter dans un court laps de temps après le retour à la conduite manuelle.

Le deuxième type de processus de réengagement, le réengagement complet après un désengagement limité, correspond à ce qui a été observé chez P1. Ce dernier présentait un engagement dans l'activité de vie à bord suffisant pour poursuivre le film. Par contre, il déclarait lui "résister " et jetait régulièrement un œil à la situation de conduite, manifestant un engagement parallèle pour la conduite.

L'activité de conduite est traditionnellement décrite avec une tâche principale (ou ensemble de tâches) de conduite et une tâche de vie à bord dite "secondaire " ou "annexe» (Forzy, 2004). Les stratégies de gestion de l'attention du conducteur, notamment visuelle, sont décrites depuis longtemps pour leurs enjeux sécuritaires (Green, 1999 pour un exemple de revue) ${ }^{10}$. Il s'agit de limiter la distraction pour ne pas impacter les boucles de coordination avec le trafic et plus encore de pilotage (aux boucles de rétroaction temporellement plus courtes).

69 Mais, plus généralement, de multiples activités peuvent avoir lieu autour de la conduite ou de divers autres sujets (radio, discussion passager, pensées privées). La gestion de ces multiples fils attentionnels est qualifiée de multitasking (Aasman \& Michon, 1992 ; Engström, Trent, \& Markulla, 2013). En ce sens, P1 fait du multitasking entre le visionnage du film et la surveillance de la scène de conduite. Avec cependant un schéma de gestion attentionnel inversé: P1 reste principalement sur le film (temporellement plus sollicitant) et jette un coup d'œil de temps en temps sur la route. C'est une rupture avec le multitasking existant aujourd'hui en conduite du fait de la disponibilité offerte par le véhicule autonome et la nature des nouvelles activités de vie à bord qu'elle autorise. L'engagement restant dans la conduite ne suffit alors plus à suivre les boucles de pilotages (nécessitant un suivi continu), mais permet de suivre celles de coordination dans le trafic, dont la temporalité est plus lente.

Ce maintien parallèle d'un engagement sur la scène routière limite l'engagement possible dans la vie à bord. Il semble cependant précieux pour le réengagement à la conduite: 1) le couplage de coordination avec le trafic est déjà présent, même fragmentaire, et non plus sacrifié comme avec P2 et 2) ce couplage ne peut que faciliter la remise en route des boucles de pilotage dont l'engagement est proche.

71 Ce fonctionnement a cependant ses limites. L'étude présentée en section précédente a mis en évidence que la motivation des participants à effectuer cette surveillance était très variable d'une personne à l'autre, et des études seraient à mener pour voir sa décroissance probable alors que la confiance dans le véhicule autonome se construit. En effet, cette boucle rémanente de coordination avec le trafic s'apparente à de la surveillance passive d'un processus, pour laquelle l'attention humaine est connue pour être peu résistante (Miller, Sun, Johns, Ive, Sirkin, Aich et al, 2015 ; Victor, Tivesten, Gustavsson, Johansson, Sangberg, \& Ljung Aust, 2018). La structure d'anticipation est maintenue pour suivre les perturbations qu'elle sélectionne comme pertinentes mais n'est pas ancrée dans l'environnement par l'usage de schèmes d'action. Ainsi, non seulement le maintien de ce double processus en parallèle chez le conducteur est coûteux cognitivement et peu robuste, mais il peut limiter la valeur d'un tel produit sur le marché, associé au temps libéré pour réaliser d'autres activités, se détendre... 
Deux pistes existent pour supporter l'engagement du conducteur sur la scène routière : de conduite avant de l'autoriser à réactiver le mode autonome, incitant ainsi le conducteur à assurer un maintien d'engagement minimal et acceptable en conduite ;

- Maintenir une sollicitation attachée à la route pour supporter l'engagement à la conduite du conducteur, par exemple par l'envoi de messages l'alertant de potentiels difficultés ou dangers environnants apparaissent pouvant impacter le trafic. De tels messages, lumineux ou sonores, pourraient être rapportés avec une intensité d'intrusivité croissante si le conducteur n'acquitte pas leur prise de connaissance.

Cependant, un véhicule autonome ne devrait pas reposer sur la responsabilité du conducteur pour détecter et corriger les limites du système. Il faudrait pour cela faire la preuve par de nouvelles études que l'attention du conducteur peut être maintenue sur la route de manière suffisamment efficace, et ce sur l'ensemble du domaine de fonctionnement de ce système.

Enfin, le troisième processus est le plus intéressant à l'usage. Il vise à favoriser un réengagement complet à la conduite malgré un désengagement fort de la conduite en phase autonome. Cela fonctionnerait également $a$ fortiori en cas de désengagement plus limité à la conduite, et permet dans tous les cas de profiter du temps à bord. Ce processus visé pourrait être rendu possible en trouvant les moyens de susciter une phase de préparation chez le conducteur en amont de son retour à la conduite manuelle. La principale limite de ce processus est qu'il nécessite que la conduite autonome soit suffisamment avancée en niveau d'autonomie (SAE niveau 4) pour offrir le temps nécessaire à la reconstruction de cette phase de préparation. Notons en effet pour rappel que la reconstruction d'une représentation de la situation de conduite dynamique a été évaluée de 10 à 20 secondes, 20 secondes permettant d'avoir intégré la vitesse relative des véhicules environnements, en plus de leur positionnement (cf. section 2.1. et $\mathrm{Lu}$, Coster, \& De Winter, 2017). Dans notre étude, les temps de réengagement n'étant que de 10 secondes, ce processus ne pouvait être observé.

En termes de pistes de conception, il s'agirait donc d'accompagner ce réengagement complet du conducteur en rendant ce dernier à la fois alerte et capable de reconstruire une conscience de la situation de conduite environnante en amont de son réengagement physique (boucle de pilotage). Nous proposons, en conclusion, une démarche en plusieurs étapes afin de favoriser: 1) lors de l'apprentissage, la constitution des schèmes adéquats à ce comportement et, 2) la possibilité de les supporter à l'usage. En supposant avoir tout le temps nécessaire, ces étapes sont représentées sur la base d'une reprise en main longue d'une minute dans le modèle d'« entonnoir de réengagement » présenté en Figure 9.

L'étape 0 « engagé dans une activité de vie à bord » correspond au moment de liberté laissé au conducteur. C'est l'espace où il peut se consacrer, une fois la confiance installée, à son activité de vie à bord. Le cockpit du véhicule est entièrement dédié à la vie à bord, tout en proposant un rappel du temps restant avant reprise de l'activité de conduite.

L'étape 1, "désengagement de la vie à bord", a pour but d'inciter le conducteur à se désengager de lui-même de ses activités pour se réintéresser à la situation de conduite. Le cockpit du véhicule ne change pas, mais les IHM embarquées présentent au conducteur la possibilité d'activer la commande pour passer à l'étape 2, tout en rappelant le retour proche à la conduite. Afin de limiter la pression, le message peut 
suivre la logique des annonces en vols dans les avions, par exemple en mettant le film en pause.

Figure $9:$ « L'entonnoir de réengagement » comme guide de conception. Figure 9: The "funnel of reengagement" as a guide for design

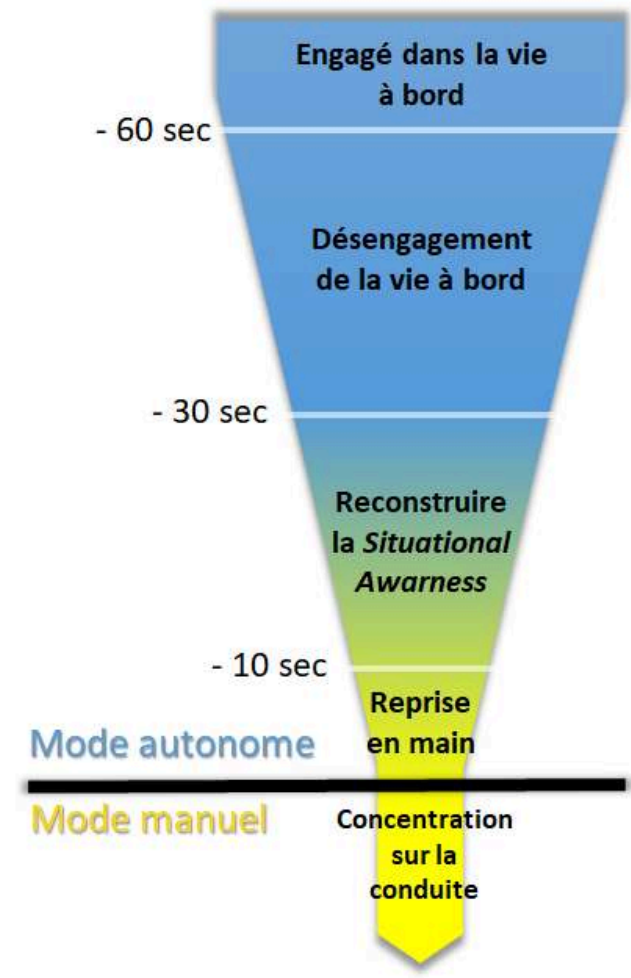

Le conducteur profite des activités de vie à bord

Le conducteur est invité à stopper son activité de vie à bord quand il le souhaite

Il est demandé au conducteur de se remettre à la conduite (posture et Situational Awareness)

Il est demandé au conducteur de reprendre le contrôle du vehicule

L'étape 2 de "reconstruction de la $S A$ » commence quand le conducteur active la commande pour sortir du mode de vie à bord ou sinon à -30 secondes. Le contenu de vie à bord disparaît. S'il s'agit d'un cockpit reconfigurable (recul et rotation des sièges), ce dernier remet le conducteur dans la position physique initiale de conduite, participant à rappeler les gestes de conduite. Enfin, des IHM sonores et visuelles (par exemple, messages vocaux explicites, IHM visuelles dont pictogrammes) peuvent inciter à regarder autour comme dans le processus de support informationnel du processus précédant.

Enfin, l'étape 3 de «reprise en main » apparaît uniquement si le conducteur ne reprend pas de lui-même la conduite manuelle avant les 10 dernières secondes du décompte. Ce décompte devient alors plus prégnant (IHM visuelle et alerte sonore) afin d'inciter toujours davantage le conducteur à reprendre la conduite en mode manuel.

Quand il sera possible techniquement, ce dernier processus permettra de cumuler à la fois les promesses technologiques de temps retrouvé pour la vie à bord, et une reprise en main supportant une adaptation complète et anticipée du couplage de conduite lors de la reprise en main. Le conducteur pourra alors s'être réengagé dans le monde de la conduite avant d'assurer le contrôle effectif de son véhicule. 


\section{BIBLIOGRAPHIE}

Aasman, J., \& Michon, J. A. (1992). Multitasking in driving. In A. Michon \& A. Akyürek (Eds.), Soar: A cognitive architecture in perspective (pp. 169-198). Dordrecht : Springer. https://doi.org/ 10.1007/978-94-011-2426-3_6

Adam, E. C. (1993). Fighter cockpits of the future. In [1993 Proceedings] AIAA/IEEE Digital Avionics Systems Conference (pp. 318-323). IEEE. https://doi.org/10.1109/dasc.1993.283529

Bainbridge, L. (1983). Ironies of automation. Automatica, 19(6), 775-779. https://doi.org/ 10.1016/0005-1098(83)90046-8

Banks, V. A., Stanton, N. A., \& Harvey, C. (2014). Sub-systems on the road to vehicle automation: Hands and feet free but not 'mind' free driving. Safety Science, 62, 505-514. https://doi.org/ 10.1016/J.SSCI.2013.10.014

Beun, C. (2015). Study on driving simulator: Effect of LoB task « watching a video » on driver behavior when taking over control in eyes off Autonomous car. Rapport Renault NT68510-2015-20.

Chalandon, X. (2007). Conscience de la situation : invariants internes et invariants externes. Thèse de doctorat en Ergonomie, sous la direction de Pierre Falzon, Paris, CNAM, 2007.

De Winter, J. C. F., Happee, R., Martens, M. H., \& Stanton, N. A. (2014). Effects of adaptive cruise control and highly automated driving on workload and situation awareness: A review of the empirical evidence. Transportation Research Part F: Traffic Psychology and Behaviour, 27, 196-217. https://doi.org/10.1016/J.TRF.2014.06.016

Dogan, E., Rahal, M.-C., Deborne, R., Delhomme, P., Kemeny, A., \& Perrin, J. (2017). Transition of control in a partially automated vehicle: Effects of anticipation and non-driving-related task involvement. Transportation Research Part F: Traffic Psychology and Behaviour, 46, 205-215. https:// doi.org/10.1016/J.TRF.2017.01.012

Endsley, M. R. (1995a). Measurement of situation awareness in dynamic systems. Human Factors, $37,65-84$

Endsley, M. R. (1995b). Toward a theory of situation awareness in dynamic systems. Human Factors, 37(1), 32-64. https://doi.org/10.1518/001872095779049543

Engström, J., Trent, V., \& Markulla, G. (2013). Attention selection and multitasking in everyday driving: A conceptual model. Driver Distraction and Inattention: Advances in Research and Countermeasures, 1, 27-54.

Forzy, J.-F. (2004). Conduite automobile et conception ergonomique. In P. Falzon (Ed.), Ergonomie (pp. 647-664). PUF : Paris.

Gibson, J. J. (1938). A theoretical field-analysis of automobile-driving. The American Journal of Psychology. Retrieved from https://www.jstor.org/stable/1416145

Gibson, J. J. (1979). The ecological approach to visual perception. Hillsdale, N. J. : Lawrence Erlbaum Associates, 1986.

Gibson, M., Lee, J., Venkatraman, V., Price, M., Lewis, J., Montgomery, O., ... Foley, J. (2016). Situation awareness, scenarios, and secondary tasks: Measuring driver performance and safety margins in highly uatomated vehicles. SAE International Journal of Passenger Cars - Electronic and Electrical Systems, 9(1), 2016-01-0145. https://doi.org/10.4271/2016-01-0145 
Gold, C., Damböck, D., Lorenz, L., \& Bengler, K. (2013). “Take over!” How long does it take to get the driver back into the loop? Proceedings of the Human Factors and Ergonomics Society Annual Meeting, 57(1), 1938-1942. https://doi.org/10.1177/1541931213571433

Green, P. (1999). Visual and task demands of driver information systems. UMTRI-98-16.

Gugerty, L. (2011). Situation awareness in driving. In D. L. Fisher, M. Rizzo, J. K. Caird, \& J. D. Lee (Eds.), Handbook of Driving Simulation for Engineering, Medicine, and Psychology (pp. 265-272). Boca Raton: CRC Press. https://doi.org/10.1201/b10836-20

Haué, J.-B. (2005). Comparing distributed cognition and course of action: an application to car driving . Proceedings of the 2005 Annual Conference on European Association of Cognitive Ergonomics .

Hutchins, E. (1995a). Cognition in the Wild. Cambridge : MIT Press.

Hutchins, E. (1995b). How a cockpit remembers its speed. Cognitive Science, 19(3), 265-288. https:// doi.org/10.1016/0364-0213(95)90020-9

Hutchins, E., \& Klausen, T. (1996). Distributed cognition in an airline cockpit. In D. Middleton \& Y. Engeström (Eds.), Communication and Cognition at Work (pp. 15-34). Cambridge : Cambridge University Press.

Le Bellu, S., \& Haué, J.-B. (n.d.). Sommes-nous prêts à conduite un véhicule autonome?

Lee, J. D., \& See, K. A. (2004). Trust in technology: Designing for Appropriate Reliance. Human Factors, 46(1), 50-80. https://doi.org/10.1518/hfes.46.1.50_30392

Leroi-Gourhan, A. (1911-1986). (2014). Le geste et la parole. [I], Technique et langage. Paris : Albin Michel.

Lu, Z., Coster, X., \& De Winter, J. (2017). How much time do drivers need to obtain situation awareness? A laboratory-based study of automated driving. Applied Ergonomics, 60, 293-304. https://doi.org/10.1016/j.apergo.2016.12.003

Lu, Z., Happee, R., Cabrall, C. D. D., Kyriakidis, M., \& De Winter, J. C. F. (2016). Human factors of transitions in automated driving: A general framework and literature survey. Transportation Research Part F: Traffic Psychology and Behaviour, 43, 183-198. https://doi.org/10.1016/J.TRF. 2016.10.007

Maturana, H. R., \& Varela, F. J. (1987). The tree of knowledge: the biological roots of human understanding. Shambhala Publications. https://doi.org/EB T MATUR

Merleau-Ponty, M. (1945). Phénoménologie de la perception. Paris : Gallimard.

Michon, J. A. (1985). A critical view of driver behavior models: What do we know, what should we do? In Human Behavior and Traffic Safety (pp. 485-524). Boston, MA : Springer US. https://doi.org/ 10.1007/978-1-4613-2173-6_19

Miller, D., Sun, A., Johns, M., Ive, H., Sirkin, D., Aich, S., \& Ju, W. (2015). Distraction becomes engagement in automated driving. Proceedings of the Human Factors and Ergonomics Society Annual Meeting, 59(1), 1676-1680. https://doi.org/10.1177/1541931215591362

Onnasch, L., Wickens, C. D., Li, H., \& Manzey, D. (2014). Human performance consequences of stages and levels of automation. Human Factors: The Journal of the Human Factors and Ergonomics Society, 56(3), 476-488. https://doi.org/10.1177/0018720813501549

Peirce, C. S. (1978). Écrits sur le signe (Vol. 31). Paris : Seuil.

Piaget, J. (1947). La psychologie de l'intelligence. Armand Colin, Vol. 249. 
Piaget, J., \& Inhelder, B. (1969). La psychologie de l'enfant. Paris : PUF.

SAE. (2018). Taxonomy and definitions for terms related to driving automation systems for on-road motor vehicles (Surface Vehicle Recommended Practice: Superseding J3016 Sep 2016).

Salembier, P., Theureau, J., Zouinar, M., \& Vermersch, P. (2001). Action/cognition située et assistance à la coopération. In $12^{\circ}$ journées francophones d'ingénierie des connaissances IC'2001. Grenoble.

Suchman, L. (1987). Plans and situated actions. The problem of human-machine communication. Cambridge : Cambridge University Press.

Theureau, J. (2006). Le cours d'action : méthode développée. Toulouse : Octarès.

Theureau, J. (2015). L'énaction et l'expérience. Toulouse : Octarès.

Vermersch, P. (1994). L'entretien d'explicitation en formation initiale et en formation continue. Paris : ESF.

Vermersch, P. (2002). La prise en compte de la dynamique attentionnelle : éléments théoriques. Expliciter, 43, 27-39.

Victor, T. W., Tivesten, E., Gustavsson, P., Johansson, J., Sangberg, F., \& Ljung Aust, M. (2018). Automation expectation mismatch: incorrect prediction despite eyes on threat and hands on wheel. Human Factors: The Journal of the Human Factors and Ergonomics Society, 60(8), 1095-1116. https://doi.org/10.1177/0018720818788164

Vygotsky, L. S. (1978). Mind in society: the development of higher psychological processes. (M. Cole, Ed.). Harvard University Press.

Zhang, B., Winter, J., Varotto, S. F., \& Happee, R. (2019). Determinants of takeover time from automated driving: A meta-analysis of 128 studies. Transportation Research Part F: Traffic Psychology and Behaviour, 64, 285-307. https://doi.org/10.1177/0018720816685832

\section{NOTES}

1. Il existe cinq niveaux d'automatisation SAE de la conduite en plus du niveau 0 . Le niveau 4 correspond à un véhicule assurant totalement la conduite sur un trajet donné.

2. "The higher they are, the farther they fall».

3. La différence entre les niveaux 4 et 5 tient à la couverture de l'infrastructure routière (une typologie de routes spécifiques pour le niveau 4 , la totalité des routes pour le niveau 5).

4. "Knowing what is going on so you can figure out what to do "

5. Cette définition de notion d'affordance est très simplifiée. La notion est objet de visions alternatives, parfois considérée comme une propriété «objective» de l'environnement indépendamment de toute perception (Gibson 1938), parfois rapprochée d'une notion de perception directe de l'environnement par l'humain.

6. Il est nécessaire dans cette perspective de faire l'hypothèse ontologique de l'existence d'une conscience préréflexive, i.e. de la possibilité qu'a le sujet d'avoir accès au sens de son activité. Le cumul de cette option sartrienne aux hypothèses énactives est exposé notamment dans Theureau (2015).

7. L'état de préparation n'est ici pas entendu forcement comme conscient et réflexif mais comment l'organisme de l'acteur maintient une préparation pour répondre à l'interaction en cours. 
8. Les temps d'expérimentation sur simulateur sont variables d'une étude à l'autre, toutefois il est coutumier d'éviter l'écriture de scénarii de plus d'une heure car ceux-ci exposent à un risque accru de cinétose.

9. Séquence où le personnage principal attend son train à la gare, quand l'immeuble d'à côté se met spectaculairement à brûler. Le héros plonge dans ce bâtiment et ressort en sauvant un caniche à trois pattes.

10. Les tableaux inspirés notamment par Zwahlen définissent des critères de sécurité en croisant la durée moyenne d'un détournement de regard avec le nombre de détournements de regard. La NHTSA (National Highway Traffic Safety Administration) s'en inspire pour définir des limites à 2 secondes de détournement de regard et à $12 \mathrm{sec}$ maximum sur le temps de détournement cumulé pour la réalisation d'une tâche donnée.

\section{RÉSUMÉS}

Avec l'arrivée du véhicule autonome, le conducteur sera amené à reprendre régulièrement la conduite d'un véhicule en déplacement et inséré dans un trafic. Ceci impliquera pour ce conducteur non seulement de reprendre le pilotage, mais aussi de se reconstruire une conscience de la situation adéquate (Situation Awareness - SA) pour évoluer en sécurité en étant capable d'anticiper les changements de trafic.

Lors d'une étude portant sur les temps de reprise en main, menée auprès de quinze utilisateurs, deux participants ont été confrontés à leurs traces d'activité lors d'épisodes de reprise en main de la conduite juste après avoir été fortement engagés dans une tâche de vie à bord. L'utilisation de la notion d'engagement développée dans le cadre du programme de recherche cours d'Action montre comment le réengagement dans la conduite se construit dynamiquement. Ces deux cas d'étude approfondie suggèrent qu'avec la pression temporelle, un conducteur novice risque d'être orienté en premier vers ce que l'environnement lui offre de plus immédiat, à savoir le pilotage du véhicule. La construction d'une conscience de la situation (SA) n'intervient que dans un second temps.

Ce moment de reprise en main et le changement radical d'orientation de l'activité qu'il implique offrent un terrain privilégié pour observer cet aspect dynamique de l'engagement. L'articulation des notions issues de la cognition située dresse un panorama des dynamiques de réengagement du couplage dans la conduite. Une typologie des processus de réengagement en est issue pour offrir des recommandations pour la conception.

With future autonomous vehicles, drivers will have to regularly take over the control of a vehicle moving in traffic. This will mean not only taking over the driving but also reconstructing a suitable awareness of the situation (Situation Awareness - SA) that enables him/her to anticipate traffic evolution.

Out of 15 participants in a study on the time needed to take over control, two were selected for an autoconfrontation interview. A detailed qualitative analysis of the driver's take over process was carried out, after they had been deeply engaged in a life-on-board task. The notion of engagement, from the Course of Action theory, shows how reengagement in driving is dynamically built. The case studies suggest that with time pressure, a novice driver is likely to be first oriented towards the immediacy of the environment, leading him to focus on the trajectory of the vehicle. Construction of situation awareness (SA) only takes place afterwards. 
This short instant of radical change in activity offers an ideal opportunity to observe this dynamic aspect of engagement. The articulation of Situated Cognition notions draws a picture of the reengagement process. The resulting typology of reengagement processes offers design principles.

INDEX

Keywords : autonomous driving, engagement, course of action, situated cognition Mots-clés : véhicule autonome, engagement, cours d'action, cognition située

\section{AUTEURS}

JEAN-BAPTISTE HAUÉ

Renault SAS, 1 avenue du Golf, 78084 Guyancourt - Jean-Baptiste.Haue@renault.com SOPHIE LE BELLU

Renault SAS, 1 avenue du Golf, 78084 Guyancourt

Université de Bordeaux, Bordeaux INP - ENSC, IMS Cognitique UMR 5218 - Sophie.LeBellu@renault.com

\section{CÉCILE BARBIER}

Consultante, associée équipe CRAFT, Université de Genève - cecile.barbier@unige.ch 